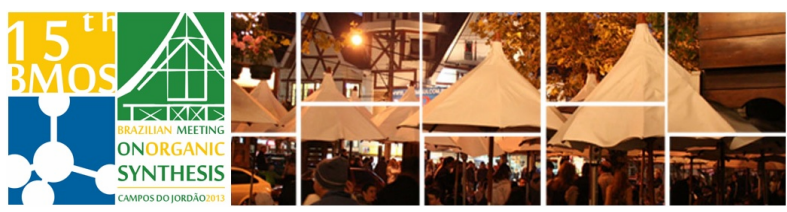

\title{
A simple access to ionic liquids using $N$-substituted imidazole derivatives from Morita-Baylis-Hillman adducts
}

\author{
Marilia S. Santos, Manoel T. Rodrigues Jr. and Fernando Coelho* \\ IQ/UNICAMP - PO Box 6154 - 13083-970 - Campinas, São Paulo, Brazil \\ *coelho@iqm.unicamp.br
}

Keywords: ionic liquids, 1,1'-carbonyldiimidazole, Morita-Baylis-Hillman adducts

\section{INTRODUCTION}

Over the years, the imidazole nucleus has attracted considerable interest due to their chemical and biological properties. Imidazole ring is part of the structure of various natural products and drugs. In chemistry are employed as ligand, catalysts for chemical reactions and also as a starting material for the synthesis of ionic liquids. ${ }^{1}$

Ionic liquids have an important role in organic synthesis, are used as solvents and catalysts, exerting a profound effect on activity and selectivity of different chemical reactions. ${ }^{2}$

In this work, we report an approach to the synthesis of ionic liquids using $\mathrm{N}$-substituted imidazole from Morita-Baylis-Hillman adducts.

\section{RESULTS AND DISCUSSION}

The study began with a synthesis of $\mathrm{MBH}$ adducts using a protocol developed by us some years ago. ${ }^{3}$

Previously, we described the synthesis of $\mathrm{N}$ substituted imidazole derivatives from Morita-BaylisHillman adducts using 1,1'-carbonyldiimidazole (CDI). The compounds were obtained in good yields and high $E$-selectivity (Scheme 1). ${ }^{4}$<smiles>[R]C(=C)C([R])O</smiles>

MBH adduct

$\mathrm{R}^{1}=$ alkyl, aryl, heteroaryl $\mathrm{R}^{2}=\mathrm{CO}_{2} \mathrm{Me}, \mathrm{CO}_{2}{ }^{t} \mathrm{Bu}, \mathrm{CN}$

Scheme 1. $\mathrm{N}$-substituted imidazole derivatives from $\mathrm{MBH}$ adducts

The $N$-substituted imidazole derivatives were treated with 1-bromobutane in acetonitrile under reflux to give the ionic liquids (Table 1 ).
Table 1: lonic liquids from $N$-substituted imidazole derivatives

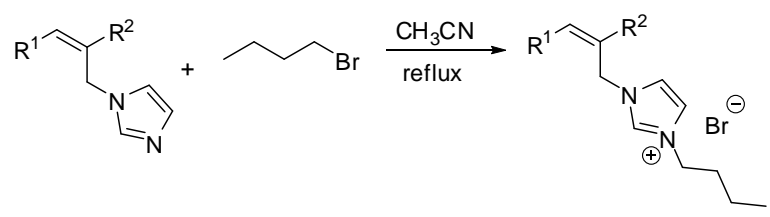

\begin{tabular}{lllc}
\hline Entry & \multicolumn{1}{c}{$\mathbf{R}^{1}$} & \multicolumn{1}{c}{$\mathbf{R}^{2}$} & Yield(\%) \\
\hline $\mathbf{1}$ & 3,4,5-OMe-Ph & $\mathrm{CO}_{2} \mathrm{Me}$ & $>95$ \\
$\mathbf{2}$ & $n$-Propyl & $\mathrm{CO}_{2} \mathrm{Me}$ & $>95$ \\
$\mathbf{3}$ & 4-Isopropyl-Ph & $\mathrm{CO}_{2}{ }^{t} \mathrm{Bu}$ & $>95$ \\
$\mathbf{4}$ & Tiazolyl & $\mathrm{CO}_{2} \mathrm{Me}$ & $>95$ \\
$\mathbf{5}$ & 4- $\mathrm{NO}_{2}-\mathrm{Ph}$ & $\mathrm{CN}$ & $45^{*}$ \\
$\mathbf{6}$ & 4-PivO-Ph & 2-Cyclopentanone & 85 \\
\hline${ }^{*}$ Yield after purification in chromatograph column &
\end{tabular}

${ }^{*}$ Yield after purification in chromatograph column

\section{CONCLUSION}

In this communication we demonstrated an easy and straightforward approach to ionic liquids synthesis using $\mathrm{N}$-substituted imidazole derivatives from $\mathrm{MBH}$ adducts. These $\mathrm{N}$-substituted imidazole compounds can be also used as platform to synthesize more complex molecules include asymmetric ionic liquids simply by performing an asymmetric reduction of the conjugated double bond.

\section{ACKNOWLEDGEMENTS}

We thank Fapesp , CNPq and CAPES for financial support

\section{REFERENCES}

${ }^{1}$ Molina, P.; Tárraga, A.; Otón, F. Org. Biomol. Chem., 2012, 10, 1711

${ }^{2}$ Lin, J.C.Y.; Huang, R.T.W; Lee, C.S.; Bhattacharyya, A.; Hwang, W.S Lin, I.J.B. Chem. Rev. 2009, 109, 3561.

${ }^{3}$ Coelho, F.; Almeida, W. P.; Veronese, D.; Lopes, E.C.S.; Silveira, G. P. C.; Rossi, R.C.; Pavam, C.H. Tetrahedron, 2002, 58, 7437.

${ }^{4}$ Santos, M.S.; Rodrigues Jr, M.T.; Santos, H.; Coelho, F.; $14^{\text {th }}$ Tetrahedron Symposium, 25-28 ${ }^{\text {th }}$ June, 2013, Vienna - Austria, A simple access to $\mathrm{N}$ substituted imidazole derivatives from Morita-Baylis-Hillman adducts using $1,1^{\prime}$-carbonyldiimidazole. 